\title{
Adrenal inclusions in congenital adrenal hyperplasia: clinical and progressive characteristics:EP-44
}

\section{INTRODUCTION}

Intra-testicular adrenal inclusions (ISIT) are benign tumors made of ectopic adrenal cortex tissue hyper stimulated by ACTH in excess.

All pathologies causing a rise ACTH levels may be associated with adrenal inclusions

They are found mainly in patients with congenital adrenal hyperplasia ( $\mathrm{CAH}$ ) untreated or poorly treated

$$
\text { AIM }
$$

Search the frequency of ISIT in CAH and specify clinical and progressive characteristics

\section{MATERIALS AND METHODS}

40 patients with CAH responsible adrenal insufficiency (35 cases in 21 and 1 in $5 \alpha$ hydroxylase) underwent testicular echo-Doppler in search of ISIT . We analysed The volume of each testicle, the presence of ISIT, their size, echogenicity and vascularity at diagnosis and during follow up
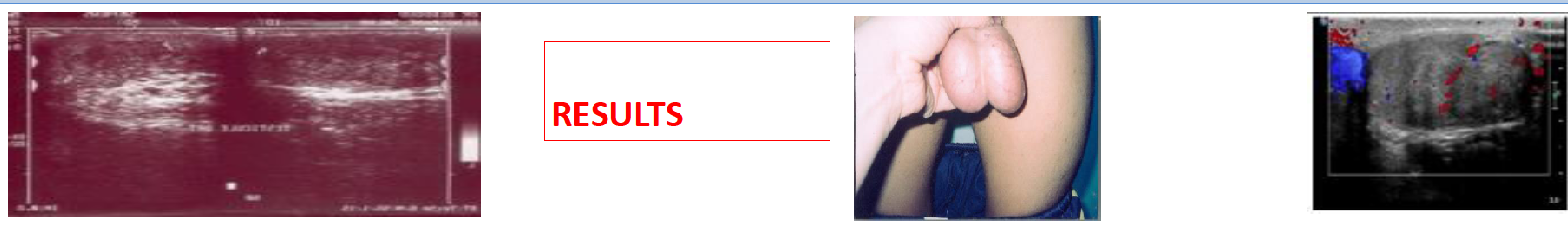

Adrenal inclusions were found in 10 patients (25\%). They were asymptomatic and bilateral in all cas. Doppler ultrasound was suggestive in all patients ( hilar localization , mitigating but not calcified appearance, vascular architecture preserved).

Partial regression after

glucocorticoid treatment was noted in $95 \%$ of cases

In two cases the adrenal inclusion continued to grow requiring orchidéctomie.

Histological study was in favor of a Leydig cell tumors

\section{ISCUSSION AND CONCLUSION}

ISIT are common in CAH(between $27 \%$ and $94 \%$ ). Related to chronic stimulation by ACTH, they often reflect an insufficient suppressive treatment with glucocorticoid. The Doppler allows the characterization . They must be systematically sought and monitored

This rare disease deserves to be known as inclusions simulate a tumor.

We must recognize their willingly hypoechoic, bilateral, symmetrical and centered hilum. The decrease in testicular volume is attributed to the increase of $17 \mathrm{OH}$ progesterone, antigonadotropic of action and may be accompanied by disruption of semen analysis.

They usually regress or stabilize with replacement glucocorticoid treatment. Sometimes they can grow, while raising the problem of the histological nature and risk of subfertility related to the mass effect of the ISIT and toxic effects of adrenal steroids and hypogonadotropic hypogonadism 